\title{
Protease resistant prion proteins are not present in sporadic "poor outcome" schizophrenia
}

\author{
Steven E Arnold, John Q Trojanowski, Piero Parchi
}

Center for

Neurobiology and

Behavior, Department of Psychiatry

S E Arnold

\section{Department of}

Neurology

$S$ E Arnold

Center for

Neurodegenerative

Disease Research,

Department of

Pathology and

Laboratory Medicine,

University of

Pennsylvania,

Philadelphia, PA, USA

J Q Trojanowski

Institute of Pathology, Division of

Neuropathology, Case

Western Reserve

University, Cleveland,

OH, USA

P Parchi

Correspondence to: Dr Steven E Arnold, 142

Clinical Research Building, 415 Curie Boulevard, Philadelphia, PA 19104, USA. Telephone 001215 573 3258; fax 001215573 2041; email:

alveus@mail.med.upenn.edu

Received 15 April 1998 Accepted 18 June 1998

\begin{abstract}
Various clinical and epidemiological data have suggested the possibility of infectious mechanisms in schizophrenia. In addition, lengthy prodromal psychiatric symptoms can presage the development of Creutzfeldt-Jakob disease, a prototypical prion disorder. Accordingly, the presence of human protease resistant prion proteins $\left(\mathrm{PrP}^{\mathrm{res}}\right)$ was assessed in postmortem frontal cortical and thalamic tissues from a prospectively accrued and well characterised sample of elderly patients with chronic, sporadic, "poor outcome" schizophrenia using a sensitive immunoblot assay. No PrP ${ }^{\text {res }}$ was found in the brains of any of the cases, providing evidence against a role for abnormal prion proteins in the pathogenesis of schizophrenia. (F Neurol Neurosurg Psychiatry 1999;66:90-92)
\end{abstract}

Keywords: schizophrenia; prion protein; CreutzfeldtJakob disease

Psychiatric symptoms are well recognised features of Creutzfeldt-Jakob disease, a prototypical prion disorder. However, several recent articles have indicated a possible relation between prion disease and severe psychiatric illness even in the absence of frank dementia and neurological signs. In an epidemiological meta-analysis of risk factors for CreutzfeldtJakob disease, Wientjens et $a l^{1}$ found that a prior history of admission to hospital for psychotic illness was 12 times more common among patients with Creutzfeldt-Jakob disease than in the general population, with the psychiatric illness predating the first "hard" signs of Creutzfeldt-Jakob disease by decades. In addition, Samaia et al described a family with a high frequency of atypical psychotic disorder associated with prominent mood disturbance and personality changes, wherein a prion protein gene mutation was found among most of the affected members. Finally, a recent analysis of new variant Creutzfeldt-Jakob disease found that most patients presented with psychiatric symptoms, including psychosis. ${ }^{3}$ Thus these reports raise the possibility that abnormal isoforms of the prion protein, such as the protease resistant form $\left(\mathrm{PrP}^{\mathrm{res}}\right)$ that has been pathogenetically linked to Creutzfeldt-Jakob disease and other prion dis- eases, may play a part in schizophrenia or schizophrenia-like psychoses.

In our prospective clinicopathological studies of schizophrenia in elderly people, we have been impressed by the frequency of profound cognitive and functional impairments suggestive of a neurodegenerative disease ${ }^{4}$ However, detailed diagnostic neuropathological examinations as well as exhaustive quantitative investigations have failed to identify even subtle evidence of neurodegenerative disease pathology or neural injury. ${ }^{5}$ Thus the basis for the deterioration in these schizophrenic patients remains unknown.

Whereas Creutzfeldt-Jakob disease is pathologically typified by widespread spongiform changes that predominantly affect the cerebral cortex, basal ganglia, thalamus and cerebellum, sensitive assays have detected $\mathrm{PrP}^{\mathrm{res}}$ in some patients with a progressive dementing disorder but no significant spongiform changes in their brains after detailed neuropathological examination. ${ }^{6-8}$ In this study, we applied a highly sensitive immunoblot technique for the detection of $\operatorname{PrP}^{\text {res }}$ in brain tissues from a sample of elderly, "poor outcome" patients with schizophrenia.

\section{Materials and methods}

Frozen frontal and thalamic tissues were obtained at postmortem from 12 patients with schizophrenia. All were participants in a prospective clinicopathological studies programme of schizophrenia ${ }^{4}$ and had been chronically in hospital with a primary diagnosis of schizophrenia that was confirmed by history and clinical examination using DSM-IV diagnostic criteria. ${ }^{9}$ The key demographic and clinical data on these patients are presented in the table. In addition to their psychiatric symptoms, eight of the patients exhibited severe and progressive cognitive and functional impairments sufficient to meet the diagnostic criteria for an additional diagnosis of dementia. Tissue blocks were dissected at postmortem and fixed in ethanol $(70 \% \mathrm{EtOH}, 150 \mathrm{mM} \mathrm{NaCl})$ or $10 \%$ neutral buffered formalin and embedded in paraffin for sectioning for diagnostic studies, while the remainder of the brain was frozen and stored at $-70^{\circ}$. Diagnostic neuropathological examinations disclosed no abnormalities in any of the subjects. In addition, one patient with neuropathologically confirmed Creutzfeldt- 


\begin{tabular}{llllllll}
\hline Patient & Age & Sex & Age of onset & $\begin{array}{l}\text { Duration of } \\
\text { illness }\end{array}$ & Dementia & Brain weight $(g)$ & PMI \\
\hline 1 & 67 & F & 16 & 51 & No & 1408 & 8.5 \\
2 & 72 & F & 24 & 48 & No & 1157 & 10.0 \\
3 & 75 & F & 23 & 52 & Yes & 1044 & 16.0 \\
4 & 75 & M & 21 & 54 & No & 1311 & 12.0 \\
5 & 76 & M & 18 & 58 & Yes & 1275 & 8.0 \\
6 & 77 & F & 39 & 38 & Yes & 1150 & 11.0 \\
7 & 77 & F & 37 & 40 & No & 1060 & 11.0 \\
8 & 80 & F & 22 & 58 & Yes & 1383 & 7.0 \\
9 & 82 & M & 22 & 60 & Yes & 1270 & 6.5 \\
10 & 87 & F & 27 & 60 & Yes & 1341 & 15 \\
11 & 89 & F & 26 & 63 & Yes & 1080 & 8.5 \\
12 & 95 & M & 25 & 70 & Yes & $1211(152)$ & $10.2(3.0)$ \\
Mean (SD) & $79.3(7.8)$ & 8F/4M & $25.0(6.8)$ & $54.3(9.2)$ & 8 Yes/4 No & 125 \\
\hline
\end{tabular}

PMI=Postmortem interval.

Jakob disease was included as a positive control.

Immunoblots were performed as previously described. ${ }^{7}$ Briefly, frozen samples of frontal cortex and thalamus were homogenised in nine volumes of lysis buffer and aliquots were digested with proteinase $\mathrm{K}(100$ or $50 \mu \mathrm{g} / \mathrm{ml}$ ) for 1 hour at $37^{\circ} \mathrm{C}$, resolved on 12 or $14 \%$ polyacrylamide gels, transferred to Immobilion $\mathrm{P}$, and incubated overnight at $4^{\circ} \mathrm{C}$ with monoclonal antibody $3 \mathrm{~F} 4$, which recognises the human PrP residues $109-112,{ }^{10}$ found in all human prion disease variants. ${ }^{11}$ Samples equivalent to $2 \mathrm{mg}$ wet tissue were loaded in lanes of the gels. The immunoblots were developed using the enhanced chemoluminescence system (ECL, Amersham, UK).

\section{Results}

Western blot analysis found a typical $19 \mathrm{kDa}$ banding pattern (PrP ${ }^{\text {res }}$ type 2 ) in the positive control case, confirming the neuropathological diagnosis of Creutzfeldt-Jakob disease (figure). By contrast, no $\operatorname{PrP}^{\text {res }}$ was found in any of the schizophrenia cases.

\section{Discussion}

Aside from the associations of psychiatric illness with the later development of frank Creutzfeldt-Jakob disease noted above, there are clinical, genetic, and epidemiological features of schizophrenia that would be compatible with a latent viral or cryptic prion disease model. ${ }^{12}{ }^{13}$ These include a high degree of heritability, regional prevalence differences, a history of urban birth and household crowding, and a history of poliomyelitis which also

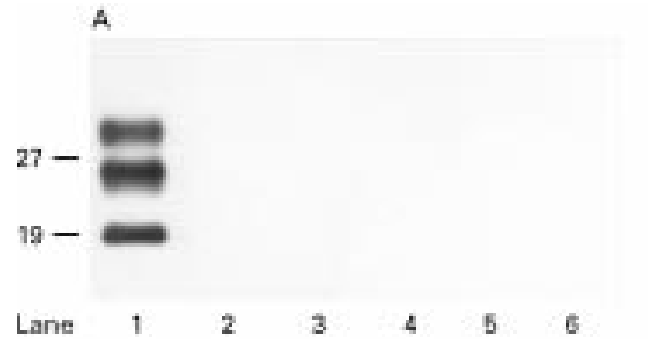

may be a risk factor for Creutzfeldt-Jakob disease. ${ }^{1}$ Furthermore, there has been increasing recognition of the cognitive impairments that are found in schizophrenia ${ }^{14-16}$ as well as the dementia that can accompany the disease in late life. ${ }^{417}$ This raises the spectre of neurodegenerative processes contributing to the disorder. Despite intensive neuropathological investigation, the defining neurobiological signature(s) of schizophrenia and its dementia remain an enigma. ${ }^{18} 19$

Our sample of elderly schizophrenic patients with poor outcome may be especially useful in the study of possible neurodegeneration or postmaturational neural injury mechanisms in schizophrenia. These patients have required continuous stay in hospital, despite numerous efforts towards deinstitutionalisation. Furthermore, most exhibited coexistent severe dementia in their final years. Thus if neurodegenerative processes are present in schizophrenia, it would be more likely in an older, severely affected sample than in a younger or better functioning group. We have expended considerable efforts to discover excessive neuropathological lesions suggesting such processes, including neuron loss, neurofibrillary and amyloid lesions, astrocytosis, ubiquitination, and microglial proliferation, but have found none. ${ }^{5}$

In this study, we used a sensitive and specific method for the detection of $\mathrm{PrP}^{\mathrm{res}}$ in a small, but very well characterised cohort of elderly schizophrenic patients. Whereas none of our cases had spongiform or other abnormal findings on neuropathological examination, this technique has been demonstrated to detect $\operatorname{PrP}^{\text {res }}$ even in the absence of such lesions. To

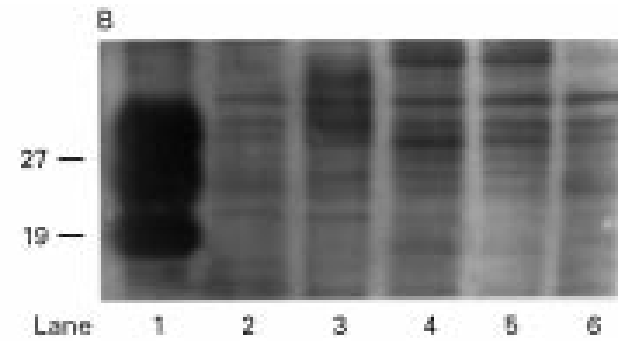

Western blot of proteinase $K$ treated brain homogenates from a patient with sporadic Creutzfeldt-fakob disease (lane 1) and from six subjects with schizophrenia (lanes 2-6), probed with the antibody 3F4. (A) Whereas the Creutzfeldt-Fakob disease sample shows protease resistant prion protein (PrP ${ }^{\text {es }}$ ) with a typical type 2 pattern, ${ }^{8}$ no $\operatorname{Pr} P^{\text {res }}$ is seen in any of the patients with schizophrenia. Prolonged exposure of the film (B) did not disclose specific PrPes peptides in the samples from patients with schizophrenia but only aspecific bands (also stained omitting the primary antibody). All samples from patients with schizophrenia were loaded 20 times more than the Creutzfeldt-fakob disease sample. 
maximise its sensitivity, we used tissue from more than one brain region, including the thalamus, a region which is consistently affected in all variants of human prion diseases. Furthermore, the $2 \mathrm{mg}$ equivalent of wet tissue that was loaded into each lane of the immunoblot gels is 20 times more than what is typically used for $\mathrm{PrP}^{\mathrm{res}}$ detection in suspected cases of prion diseases. Our failure to detect $\mathrm{PrP}^{\mathrm{res}}$ by immunoblot provides further evidence against the presence of abnormal prion proteins as an infectious aetiology in schizophrenia.

We thank Dr Pierluigi Gambetti, and the residents and staff of the University of Pennsylvania's Schizophrenia MHCRC and the Department of Pathology and Laboratory Medicine for thei ssistance in this study. We also express special appreciation to the patients and their families whose generosity made this research possible. This work was supported by grants from the AG08155).

1 Wientjens DPWM, Davinpour DVM, Hofman A, et al. Risk factors for Creutzfeldt-Jakob disease: a re-analysis of casefactors for Creutzfeldt-Jakob disease: a re-an
control studies. Neurology 1996;46:1287-91.

2 Samaia HB, Mari JdJ, Vallada HP, et al. A prion-linked psySamaia HB, Mari JdJ, Vallada HP, et al.
chiatric disorder. Nature 1997;390:241.

3 Zeidler M, Johnstone EC, Bamber RW, et al. New variant Creutzfeldt-Jakob disease: psychiatric features. Lancet 1997;350:908-10

4 Arnold SE, Gur RE, Shapiro RM, et al. Prospective clinicopathological studies of schizophrenia: accrual and assessment. Am F Psychiatry 1995;152:731-7.

5 Arnold SE, Trojanowski JQ, Gur RE, et al. Absence of neurodegeneration and neural injury in the cerebral cortex in a sample of elderly patients with schizophrenia. Arch Gen Psychiatry 1998;55:225-32.
6 Castellani R, Parchi P, Stahl J, et al. Early pathologic and biochemical changes in Creutzfeldt-Jakob disease: a study of brain biopsies. Neurol 1996;46:1690-3.

7 Parchi P, Castellani R, Cortelli P, et al. Regional distribution of protease-resistant prion protein in fatal familial insomnia. Ann Neurol 1995;38:21-9.

8 Capellari S, Vital C, Parchi P, et al. Familial prion disease with a novel 144-bp insertion in the prion protein gene in a Basque family. Neurolology 1997;49:133-41.

9 American PA. Diagnostic and statistical manual of mental disorders. 4th ed. Washington, DC: American Psychiatric Association, 1994

10 Kascsak RJ, Rubenstein R, Merz PA, et al. Mouse polyclonal and monoclonal antibody to scrapie-associated fibril proteins. F Virol 1987;61:3688-93.

11 Parchi P, Castellani R, Capellari S, et al. Molecular basis of phenotypic variability insporadic Creutzfeldt-Jakob disease. Ann Neurol 1996;39:767-78.

12 Yolken RH, Torrey EF. Viruses, schizophrenia, and bipolar disorder. Clin Microbiol Rev 1995;8:131-45.

13 Squires RF. How a poliovirus might cause schizophrenia: a commentary on Eagles' hypothesis. Neurochem Res 1997; 22:647-56.

14 Saykin AJ, Shtasel DL, Gur RE, et al. Neuropsychological deficits in neuroleptic naive patients with first-episode schizophrenia. Arch Gen Psychiatry 1994;51:124-31.

15 Waddington JL, Youssef HA. Cognitive dysfunction in chronic schizophrenia followed prospectively over 10 years and its longitudinal relationship to the emergence of tardive dyskinesia. Psychol Med 1996;26:681-8.

16 Gold JM, Weinberger DR. Cognitive deficits and the neurobiology of schizophrenia. Curr Opin Neurobiol 1995;5:225 30.

17 Davidson M, Harvey PD, Powchik P, et al. Severity of symptoms in chronically institutionalized geriatric schizophrenic patients. Am f Psychiatry 1995;152:197-207.

18 Arnold SE, Trojanowski JQ. Recent advances in defining the neuropathology of schizophrenia. Acta Neuropathol 1996; 92:217-31.

19 Harrison PJ. On the neuropathology of schizophrenia and its dementia; neurodevelopmental, neurodegnerative, or both. Neurodegeneration 1995;4:1-12. 